\title{
Maintenance Scheduling of Distribution System with Optimal Economy and Reliability
}

\author{
Siyuan Hong, Haifeng Li, Fengjiao Wang \\ School of Electrical Power, South China University of Technology, Guangzhou, China \\ Email: scuthsy@163.com
}

Received June 2013

\begin{abstract}
With the continuous expansion of power distribution grid, the number of distribution equipments has become larger and larger. In order to make sure that all the equipments can operate reliably, a large amount of maintenance tasks should be conducted. Therefore, maintenance scheduling of distribution network is an important content, which has significant influence on reliability and economy of distribution network operation. This paper proposes a new model for maintenance scheduling which considers load loss, grid active power loss and system risk as objective functions. On this basis, Differential Evolution algorithm is adopted to optimize equipment maintenance time and load transfer path. Finally, the general distribution network of 33 nodes is taken for example which shows the maintenance scheduling model's effectiveness and validity.
\end{abstract}

Keywords: Maintenance Scheduling; Multi-Objective; Differential Evolution Algorithm; Condition Based Maintenance

\section{Introduction}

With the continuous development of distribution network, maintenance scheduling of distribution equipments has become an important work of distribution network operation dispatching. Making scientific and reasonable maintenance plan is beneficial to enhance the reliability of power distribution system operation. Besides, it can improve the management level and economic benefits of Power Supply Company.

In actual work, maintenance scheduling of distribution network is arranged artificially, according to the experience of power department, which is checked by operating crew to make sure the stability of power distribution system. However, this arrangement method only focuses on the security of distribution system, while neglects the economical efficiency. Instead of artificial scheduling, maintenance scheduling should be an optimized process based on scientific and effective mathematical model, which can avoid the subjectivity and randomness of maintenance.

The study of maintenance scheduling generally optimizes the objective function of economic index by different optimization algorithms. Reference [1] introduces an automatic scheduling method using heuristic algorithm to obtain the optimal switching combination, which considers load loss and switch operation cost as the objective functions. Reference [2] puts forward an im- proved Genetic Algorithm (GA) using infeasible degree to retain the good genes on infeasible solution, and shows that the optimization procedure has less chance to get into local convergence. On the other hand, reliability index is also an important factor of maintenance scheduling. Recently, Power Supply Company has conducted pilot application of the Reliability Centered Maintenance (RCM) [4], in reference [5], its optimization objective is to lower the value of Expected Energy Not Supplied (ENNS) and improve the power system's reliability.

However, for the maintenance scheduling problem of distribution system, the reliability and economy of distribution network operation should be both considered as the optimization objective functions. This paper makes a comprehensive analysis of objective functions, including load loss, grid active power loss and system risk. Then, establish a multi-objective mathematical model with the three objective functions above. In the following, the method of using Differential Evolution algorithm to optimize the maintenance scheduling is proved to be effective and feasible by the example.

\section{Maintenance Scheduling Model}

\subsection{Multi-Objective Optimization Model}

General maintenance scheduling optimization problem will integrate several objective functions into single one by the weighting method as follow: 


$$
\begin{gathered}
\min \sum_{i=1}^{n} \lambda_{i} f_{i}(x) \\
\text { s.t. } g_{j}(x) \leq 0, j=1,2 . . l \\
z_{h}(x)=0, h=1,2 . . m
\end{gathered}
$$

where $x$ is the maintenance time vector, $\lambda_{i}$ is the weight of optimize objective function $i, l$ is the total number of inequality constraints, $m$ is the total number of equality constraints.

However, weighting single objective optimization method has the following defects:

- Enough prior knowledge is required to determine the weight of each objective function.

- Only one Pareto optimal solution can be obtained in each optimization time, which is difficult to judge the reliability and optimality of the optimization results.

- Each objective function has different dimension.

- Considering about all these, this paper adopts multi-objective optimization model to optimize several objective functions and requires that all objective functions meet the condition of setting constraints, which is shown as follow:

$$
\begin{aligned}
& \operatorname{Min}_{X \in R} F(X)=\left(f_{1}(X), f_{2}(X) \ldots, f_{k}(X)\right) \\
& \text { s.t. } g(X)=\left(g_{1}(X), g_{2}(X), . \quad g_{m}(X)\right) \leq 0 \\
& R=\{(X \mid g(X) \leq 0\} \\
& X=\left(x_{1}, x_{2} . . X_{n}\right), X \in R
\end{aligned}
$$

where $F(X)$ is the optimization target vector, $g(X)$ is the constraint vector, $X$ is the decision variable.

\subsection{Optimization Objective Functions}

The purpose of arranging maintenance scheduling is not only to transfer load as much as possible, but to consider the economy and reliability of distribution network operation. Therefore, maintenance scheduling of distribution is a combinatorial optimization problem of multi-objective and multi-constraint, which is related to the objective functions including the following aspects:

1) Load Loss

$$
f_{1}=\operatorname{Min}\left(\lambda \times \sum_{i \in N} P_{i} \times T_{i}\right)
$$

where $\lambda$ denotes average electricity price, $N$ means the assemblage of transfer nodes, $P_{i}$ is the load loss, $T_{i}$ is the maintenance continuous time.

2) Grid Active Power Loss

In order to avoid the outage of distribution network caused by equipment maintenance, we should conduct the network load transfer and besides, choose the optimal transfer path to reduce the grid active power loss, which is the target of load transfer in equipment maintenance.

$$
f_{2}=\operatorname{Min}\left(\sum_{k \in M} \Delta P_{k}\right)
$$

where $\Delta P_{k}$ denotes the grid active power loss of transfer path $k, M$ denotes the assemblage of all transfer paths.

3) System Risk

Generally, maintenance scheduling optimization model requires only that transfer strategy meet the network power flow constraint, seldom considering the problem of load equalization. The risk value of power distribution system is calculated as follow:

$$
f_{3}=\operatorname{Min}\left(\sum_{j=1}^{n} P_{j} \sum_{e=1} R_{e}\right)
$$

where $P_{j}$ denotes the load of node $j, R_{e}$ is the failure rate of main equipments on transfer path.

The risk assessment value can be divided into three levels as "Low Risk”, "Medium Risk” and "High Risk”, corresponding to evaluation score 0 - 0.3, $0.3-0.7,0.7$ 1.0 respectively.

The selection of power load transfer paths is closely related to the reliability of transfer line. If the power load of maintenance line is transferred to another line of low reliability in distribution network, the failure risk of transfer line will greatly increase, which will impact the reliable operation of distribution network. Therefore, we should conduct the calculation of line risk and transfer the power load to a high reliability line as far as possible.

In this paper, combining with Condition Based Maintenance (CBM) conducted by Power Supply Company, the health status of distribution equipments on transfer path are evaluated and then, make a prediction of equipment failure rate according to the health evaluation results. After that, Per-unit value of the line load is calculated based on the max line load. In the following, system risk is calculated and the level of risk assessment is set up according to results of risk value.

\subsection{Health State and Failure Rate}

Health evaluation is a comprehensive evaluation process, which means that the electrical equipment's health state is evaluated by various state parameters, according to the health state, the hidden defects of equipment are found out in time and Power Supply Company can conduct the maintenance to make sure that the equipment is in healthy condition [6].

This paper adopts Fuzzy Variable Weight Analysis method to evaluate the health degree of distribution equipments. The method can adjust the weights of equipments' state parameters automatically according to the relationship and quality of different parameters. The procedure of distribution equipments' health evaluation can be described as follows: 


$$
\begin{aligned}
b_{i} & =\sum_{j=1}^{m} W_{i j} \circ R_{i j} \\
B & =\left[b_{1}, b_{2}, \ldots, b_{s}\right] \\
Y & =\sum_{f=1}^{s} c_{f} b_{f}
\end{aligned}
$$

where $R_{i j}$ denotes the fuzzy evaluation score and $A_{i j}$ denotes the weight of each evaluation state parameter. $B$ denotes the fuzzy membership matrix of evaluation result. Formula (8) is the weighted summation formula; $c_{f}$ is the values of different evaluation indexes.

In practical evaluation, when some state parameters of power equipments are in extremely serious status, we can adjust the parameters' weights by using equilibrium coefficient. The variable weight formula is as follow:

$$
W\left(x_{i}\right)=w_{i o} x_{i}^{1-a} / \sum_{i=1}^{n} w_{i o} x_{i}^{1-a}
$$

where $X_{i}$ denotes the value of each state parameter, $W_{\text {io }}$ denotes the fixed weight of each parameter. $a$ is the equilibrium coefficient which values between 0 and 1 . After that, refer to the EA general formula of risk and failure probability, the fault rate formula of equipment health state is as follow:

$$
\lambda(Y)=K e^{C Y}
$$

where $K$ denotes the proportionality coefficient and $C$ denotes the curvature coefficient, which are calculated according to the statistics of equipments' health state and failure probability in the region.

\section{Differential Evolution Algorithm}

Differential Evolution (DE) algorithm is an effective and robust method, which is used to solve the complex functions with the Characteristics of Non-linear, non-differrentiable and high-dimensional. At present, DE algorithm has been widely used by the experts and scholars in different research field, which has become an important branch of Evolutionary Algorithm (EA).

DE algorithm adopts the searching strategy of "greed", which means after operation process of mutation and crossover, test individual $u_{i}^{t+1}$ can become the next generation only when its fitness is better than the original individual $x_{i}^{t}$. Otherwise, $x_{i}^{t}$ is regarded as the next generation. In this process, completely dominate $\left(u_{i}^{t+1} \prec\right.$ $\left.x_{i}^{t}\right)$ based on the concept of Pareto theory dominant is used among the chosen operation.

In addition, another feature of DE algorithm is adopting elitism strategy to keep the diversity of final solutions. The reason is that we can't guarantee that the Pareto optimal solution of current generation is always the optimal in each generation. Therefore, we use an external storage to store the Pareto optimal solutions which have been found starting from the initial population and compete the distance between the current solutions and the solutions in the storage.

\section{Example Analysis}

This paper optimizes a maintenance schedule of the general 33 nodes distribution network [11], which uses the presented method in this paper. The distribution system network is shown as the Figure 1.

Based on the method above, the maintenance week scheduling of two lines on 33 nodes network is arranged. The parameters of objective functions are set as Table $\mathbf{1 .}$

Using Differential Evolution algorithm to optimize the maintenance scheduling, the parameters of Differential Evolution algorithm are set as Table 2.

The optimization results are shown as Table $\mathbf{3}$.

In Table 3, we can see that when system risk is considered as objective function (Strategy 1), the grid active power loss is more than but close to that in the situation without considering (Strategy 2) and that, all network loads can be transferred by contact switch operation.

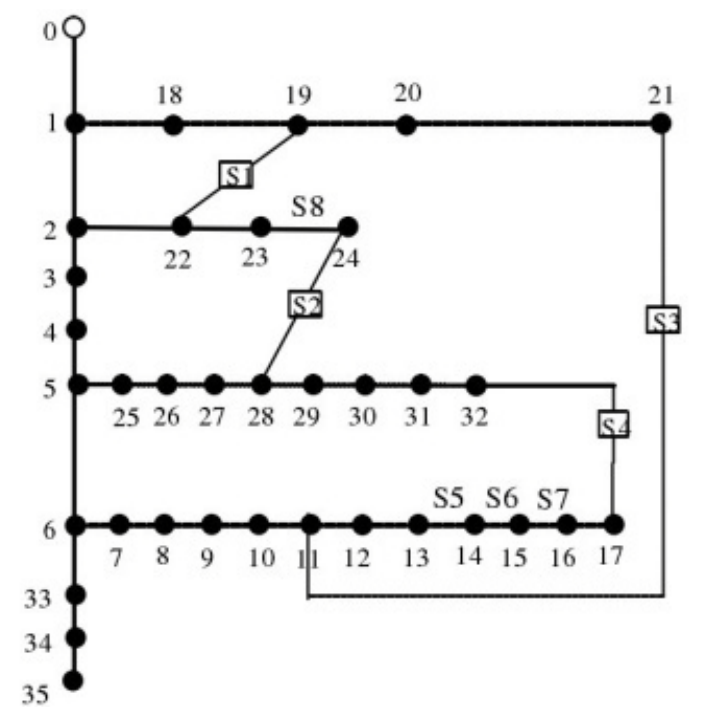

Figure 1. Distribution network of 33 nodes.

Table 1. Parameters setting of objective functions.

\begin{tabular}{ccc}
\hline \multirow{2}{*}{ Parameters Setting of Objective Functions } & Parameters Setting \\
\cline { 2 - 3 } & Average Price & The Maintenance Time \\
\hline Parameters Value & 0.5 RMB/kWh & 1 hour \\
\hline
\end{tabular}


Table 2. Parameters setting of algorithm.

\begin{tabular}{cccccc}
\hline \multirow{2}{*}{ Parameters Setting of Algorithm } & \multicolumn{4}{c}{ Parameters Setting } \\
\cline { 2 - 4 } & \multicolumn{2}{c}{ Parameter of Crossover } & Parameter of Mutation & \multirow{2}{*}{ Population Size } & Maximum Generation \\
\cline { 2 - 4 } & CR min & CR max & $\boldsymbol{F}_{\mathbf{0}}$ & & \multirow{2}{*}{000} \\
\hline Parameters Value & 0.1 & 0.9 & 0.8 & 50 \\
\hline
\end{tabular}

Table 3. Results of optimization.

\begin{tabular}{|c|c|c|c|c|c|}
\hline \multirow{2}{*}{ Results of Optimization } & \multicolumn{5}{|l|}{ Objective Function } \\
\hline & Maintenance Time & Contact Switch Operation & Load Loss & Grid Power Loss & System Risk \\
\hline $\begin{array}{l}\text { Considering LINE RISK } \\
\text { (Strategy 1) }\end{array}$ & $\begin{array}{l}\text { Wed 8:00 } \\
\text { Wed 14:00 }\end{array}$ & $\begin{array}{l}\text { Close } \mathrm{S}_{2} \\
\text { Close } \mathrm{S}_{3} \mathrm{~S}_{4} \\
\text { Open } \mathrm{S}_{5}\end{array}$ & 0 & 0.0093 & 0.3090 \\
\hline $\begin{array}{l}\text { Without Considering LINE RISK } \\
\text { (Strategy 2) }\end{array}$ & $\begin{array}{l}\text { Wed 8:00 } \\
\text { Mon 8:00 }\end{array}$ & $\begin{array}{l}\text { Close } \mathrm{S}_{2} \\
\text { Close } \mathrm{S}_{3} \mathrm{~S}_{4} \\
\text { Open } \mathrm{S}_{6}\end{array}$ & 0 & 0.0079 & 0.6087 \\
\hline
\end{tabular}

However, as the table shown, the system risk (Medium Risk) of the second strategy is much higher than the system risk (Low Risk) of strategy 1 because the transfer line 1 - 18 is in the state of high system risk. The failure rate of transfer line will greatly increase and lead to the higher system risk if the power load is transferred to line. Therefore, according the optimization results, line risk degree ought to be taken into account in the load transfer path selection of maintenance scheduling.

\section{Conclusions}

This paper establishes a multi-objective optimization model of distribution equipments' maintenance scheduling, considering the functions of load loss, grid active power loss and line risk. Through the analysis of distribution network system of 33 nodes, conclusions are drawn as follows:

1) Combining with the Condition Based Maintenance and risk assessment of distribution network equipments, this paper puts forward to consider system risk as optimization objective function, by evaluating equipments' health state, forecasting equipment failure rate and system risk, in order to avoid the disadvantage of overload operation.

2) Based on the theory of Pareto domination, a multiobjective optimization mathematical model is established according to the practical situation of maintenance scheduling. The diversity validation can keep the diversity of the Pareto optimal solutions, which is an effective method of solving multi-objective problem and making maintenance plan.

3) The optimization results of maintenance scheduling provide a feasible maintenance scheduling and transfer path, which greatly improve the reliability and economy of power system's operation and have theoretical and practical significance.

\section{Acknowledgements}

This work is supported by High-tech Industrialization Key Project in Guangdong Province (No. 2010A010200005).

\section{REFERENCES}

[1] X. Y. Mo, "Research of Optimal Load Transfer Path for Distribution Network Maintenance,” Master Thesis, North China Electric Power University, China, 2006.

[2] X. C. Huang, "Research on Maintenance Scheduling Problem of Distribution Network," Ph.D. Thesis, North China Electric Power University, China, 2007.

[3] T. Sawa, T. Furukawa, M. Nomoto, T. Nagasawa, T. Sasaki, K. Deno and T. Maekawa, "Automatic Scheduling Method Using Tabu Search for Maintenance Outage Tasks of Transmission and Substation System with Network Constraints," IEEE Power Engineering Society 1999 Winter Meeting, Vol. 2, 1999, pp. 895-900. http://dx.doi.org/10.1109/PESW.1999.747287

[4] J. X. Huang, Y. P. Bian and W. S. Cai, "Equipment Maintenance Decision-making Research Based Improved RCM Analysis,” Quality, Reliability, Risk, Maintenance, and Safety Engineering (ICQR2MSE) International Conference, 2012, pp. 487-490.

[5] D. F. Zhao, X. F. Duan and L. Zhang, "Maintenance Scheduling of Power Transmission Equipment Considering Equipment Condition and System Risk, "Journal of Xi'an Jiaotong University, Vol. 46, No. 3, 2012, pp. 8489.

[6] J. J. Huan, G. Wang and H. F. Li, "Risk Assessment of XLPE Power Cables Based on Fuzzy Comprehensive Evaluation Method,” Power and Energy Engineering Conference (APPEEC), 2010, pp. 1-4.

[7] P. Duan, "Evaluation the Impacts of System Load Demands and Load Rates on System Reliability Perfor- 
mance," Ph.D. Thesis, Chongqing University, China, 2012.

[8] N. M. Tabari and S. N. Mahmoudi, "Maintenance Scheduling Aided by a Comprehensive Mathematical Model in Competitive Environments," International Conference on Power System Technology-Power, Singapore, 2004, pp. 1774-1779.

[9] J. Wang and F. S. Wang “Optimal Maintenance Strategies for Generation Companies in Electricity Markets with Risk Management,” Transmission and Distribution Con- ference \& Exhibition, Asia and Pacific Dalian, China, 2005, pp. 1-5.

[10] L. H. Wu, "The Research and Applications of Differential Evolution Algorithm,” Master Thesis, Hunan University, China, 2007.

[11] M. E. Baran and F. F. Wu, "Network Reconfiguration in Distribution Systems for Loss Reduction and Load Balancing,” IEEE Transactions on Power Delivery, Vol. 4, No. 2, 1989, pp. 1401-1414.

http://dx.doi.org/10.1109/61.25627 